\title{
METODOLOGÍA DE TIPOS IDEALES \\ CON DATOS CATEGÓRICOS. EL ANÁLISIS \\ DEL MERCADO LABORAL JUVENIL
}

\author{
POR \\ JOSÉ MIGUEL SANTOS PRECIADO
}

\section{Introducción}

La problemática de la medición en la ciencia geográfica ha estado condicionada, desde siempre, por la artificiosidad que caracteriza a la delimitación de los elementos base de la investigación. El empleo de unidades espaciales como individuos propios de nuestra disciplina supone la sustitución de datos individuales, de personas u otros atributos geográficos directos, por unas nuevas entidades espaciales, establecidas, las más de las veces, sin criterios geográficos definidos. Esta circunstancia produce el paso de magnitudes medidas en escala nominal (e. g. el sexo, estado civil, edad, de personas) a una escala de medida cuantitativa (porcentaje de nombres o mujeres de un grupo de edad determinado, referidos a una unidad territorial administrativa), originando la denominada falacia ecológica.

Las alternativas metodológicas más comúnmente adoptadas para la solución del problema han sido de dos tipos (Bosque Sendra, J.; Chuvieco Salinero, E. y Santos preciado, J. M., 1983). Desde una perspectiva fundamentalmente geográfica, se trataría de la definición, previa a cualquier trabajo de investigación, de individuos geográficos que

José Miguel Santos Preciado. Universidad Nacional de Educación a Distancia.

Estudios Geográficos

Tomo LX, n. ${ }^{\circ} 236$, julio-septiembre 1999 
se adecuaran a los objetivos del estudio (Muguruza Cañas, C. y Santos Preciado, J. M., 1989). La segunda vía de análisis plantea una metodología basada en la utilización de datos individuales, medidos sobre objetos naturales y no arbitrarios, siguiendo el análisis de datos categóricos (Agresti, A., 1990).

Desde esta segunda perspectiva de investigación, planteamos en el presente trabajo, la elaboración de una metodología específica para el tratamiento de la problemática laboral juvenil, a partir de datos categóricos obtenidos mediante encuesta. El método invierte el orden clásico en el tratamiento de la información recogida. En vez de definir previamente los entes espaciales, como soportes geográficos de la observación, para, a continuación, referir los atributos y variables a estas unidades de análisis, se trataría de clasificar el colectivo de los jóvenes en «tipos», homogéneos desde el punto de vista de su problemática laboral específica. Esta tipología, elaborada a partir de los atributos medidos en escala categórica, permitiría la identificación de tipos ideales desde los que reconstruir los procesos sociales y de diferenciación espacial consecuentes.

\section{Metodología de análisis de la problemática laboral juvenil} a partir de la definición de tipos ideales

Planteamiento del problema.-El mercado de trabajo juvenil puede ser considerado como un segmento del mercado laboral general con unas características propias. Pero incluso desde esta perspectiva, no constituiría un todo unitario. La fragmentación social y laboral de nuestro mundo afecta a los jóvenes, según sectores de población diferentes. Así, junto a jóvenes en paro o con trabajos en precario, sobreviviendo de la economía sumergida, se hallan otros con empleos estables y bien remunerados o jóvenes empresarios con capacidad e iniciativa para crear empresas de éxito social. Diversas variables resultan fundamentales en la intención de delimitar submercados laborales - tipo, integrados por jóvenes con una problemática laboral homogénea. Estos tipos ideales, construidos según el criterio de investigación sociológica de A. Weber (Jiménez Blanco, J., 1978), tienen sentido como arquetipos de la realidad social que intentan representar. 
Un conjunto de variables elementales: sexo, edad, nivel de instrucción, relación con la actividad económica (ocupado/parado) y cualificación del trabajo realizado (en el caso de estar ocupado) pueden servir de base para obtener una aproximación a los «tipos de jóvenes» más representativos frente al mercado laboral. Conviene, sin embargo, recordar, que cualquier metodología que trate de identificar tipos ideales reduce la complejidad del mundo existente a estereotipos que facilitan su comprensión, aunque se pierda parte de la información. En la realidad, se da todo tipo de transiciones entre los grupos representados, pero la economía del análisis obliga a estas renuncias menores (Eusya, 1988).

Metodología estadística de construcción de tipologías con datos categóricos. - El tratamiento tradicional que se ha seguido en el análisis de las relaciones entre dos o más variables de tipo cualitativo ha sido el de construir una tabla de contingencia (Sánchez Carrión, J. J., 1984). La tabla de contingencia distribuye el colectivo de personas objeto de estudio en las casillas resultantes del cruce de categorías de diversas variables. El número de variables empleadas es optativo, originando tablas de dos, tres, ... y, en general, n dimensiones.

La metodología que proponemos intenta seleccionar, en una tabla de contingencia, definida por las variables o dimensiones que se consideren fundamentales, exclusivamente, aquellas celdas que tienen un mayor grado de representatividad. Para definir estas casillas, de superior peso relativo, se comparan los valores reales del análisis con los obtenidos de la hipótesis de independencia, destacando los valores que superan la unidad.

$$
\frac{f_{i j k l}}{f_{i j k l(\text { esperado })}}>1
$$

Los valores esperados, obtenidos de la condición de independencia total, se obtendrían considerando que la frecuencia conjunta sería igual al producto de las frecuencias relativas marginales unidimensionales (Ruiz-Maya Pérez, L. et alii, 1990). A modo de ejemplo, en el supuesto de una tabla cuatridimensional, obtenida por la intersección de cuatro 
variables, podríamos expresar la relación de independencia entre vaiables por la educación:

$$
f_{i j k l(\text { esperado })}=\left(\frac{f_{i 0000}}{f_{0000}} \cdot \frac{f_{0 j 00}}{f_{0000}} \cdot \frac{f_{00 k 0}}{f_{0000}} \cdot \frac{f_{0000}}{f_{0000}}\right) \times f_{0000}
$$

siendo, en general, la frecuencia marginal de tres variables, i, j y k:

$$
f_{000 l}=\sum_{i=1}^{I} \sum_{j=1}^{J} \sum_{k=1}^{K} f_{i j k l}
$$

y el total de todas las celdas:

$$
f_{0000}=\sum_{i=1}^{I} \sum_{j=1}^{J} \sum_{k=1}^{K} \sum_{l=1}^{L} f_{i j k l}
$$

El tratamiento restringido de las casillas de mayor peso relativo permite avanzar en la reconstrucción de tipos genéricos de individuos, representativos del colectivo que se desea analizar, agrupando aquellas combinaciones de categorías entre variables que mantienen una analogía o afinidad superior. La metodología teórica propuesta se comprende mejor en su aplicación a una problemática particular, como la que nos ocupa.

La delimitación de los tipos de jóvenes según la metodología anterior.-La utilización de la metodología para la definición de los «tipos ideales de jóvenes», respecto a su incorporación al mercado laboral, la hemos realizado sobre la información obtenida en un trabajo de campo de reciente ejecución, efectuado con encuesta muestral cumplimentada a 542 jóvenes, de edad comprendida entre 15 y 29 años, que habiendo abandonado el sistema educativo reglado residieran en la periferia sur de Madrid (Aguilera Arilla, M. J. et alii., 1996). El estudio consistía en una análisis exhaustivo de las características socio-demográficas, nivel de formación adquirido e integración y valoración del mercado de trabajo de la zona de residencia. La dificultad de interpretar las respuestas 
globales, representadas en 149 variables, codificadas en escala nominal, nos sugirió la posibilidad de clasificar previamente la información con objeto de facilitar el análisis.

En esencia, la vía metodológica utilizada ha consistido en definir un reducido número de variables categóricas «n», fundamentales en el estudio, en la perspectiva de representar la distribución de la población juvenil en la tabla de contigencia n-dimensional resultante. Se trataría de comparar, en las casillas del cruce de categorías múltiple, los valores observados en la realidad con los valores esperados de una situación de independencia entre las variables, con la intención de conservar, exclusivamente, la información de aquellas casillas que se alejaran de la situación de azar y fueran el resultado de la interacción entre las variables. Veamos más detenidamente las diversas fases de la investigación realizada.

Definición de las variables representativas del fenómeno anali$z a d o$. - Las variables seleccionadas son: sexo, edad, nivel de instrucción, situación respecto a la actividad (parado/ocupado) y nivel de salario. Todas las variables se hallaban codificadas en escala categórica habiendo, incluso, reducido el número de clases de cada una de ellas por los motivos antes apuntados. Las categorías y significado de las variables vienen recogidas en el cuadro I.

La tabla de contingencia, elaborada con las variables sexo, edad, nivel de estudio y salario (esta variable incluye, en realidad, a la situación

CUADRo I

VARIABLES DEL ANÁLISIS Y SUS CATEGORÍAS

\begin{tabular}{lll}
\hline Variables & Categorías & Significado \\
\hline Sexo & 1 y 2 & $1=$ hombre. $2=$ mujer \\
Edad & 1,2 y 3 & $1=15-19$ años. $2=20$ - 24 años. $3=25-29$ años. \\
Nivel de estudios & 1,2 y 3 & $1=$ primaria. $2=$ F.P. y B.U.P. $3=$ universitario. \\
Actividad & 1 y 2 & $1=$ parado. $2=$ ocupado. \\
Salario & $1,2,3,4$ y 5 & $1=$ sin salario. \\
& & $2=$ inferior a 50.000 pts. \\
& $3=$ entre 50.000 y 100.000 pts. \\
& $4=$ entre 100.000 y 150.000 pts. \\
& $5=$ superior a 150.000 pts. \\
\hline
\end{tabular}

Fuente: E. P. 
respecto a la actividad, al representar la clase 1 a los jóvenes en paro) contendría un total de 90 casillas, resultado del producto del número de categorías parciales de cada variable $(2 \times 3 \times 3 \times 5=90)$.

Construcción de los tipos ideales, a partir de la identificación de las combinaciones de categorías más representativas.-De la asociación existente entre las variables, cabe esperar una concentración superior de jóvenes en algunas de las 90 casillas, precisamente en aquellas en las que las combinaciones de sus categorías comienzan a perfilar los tipos más representativos. Así, mientras parece lógica la combinación : sexo $=1$, edad $=1$, nivel de estudios $=1$, salario $=1$, como correspondiente a un joven varón de edad inferior a 20 años, que ha abandonado los estudios sin un grado de formación idóneo y que se encuentra en el paro, parecía menos lógica la combinación: $\operatorname{sexo}=1$, edad $=1$, nivel de estudios $=1$, salario $=4$, representativa del mismo joven trabajando con un alto salario.

Esta breve reflexión nos ofrece la pauta de la metodología a aplicar. Se trata de comparar para las 90 casillas posibles, los valores observados en la realidad con los valores esperados según la hipótesis de la independencia de las variables. Para ello, hemos obtenido la relación existente entre ambas frecuencias (observada y esperada), destacando, únicamente, los valores que quedan por encima de la unidad. Veamos el cálculo realizado para una de las combinaciones de categorías posibles.

Si nos refiriéramos a un caso particular $(\operatorname{sexo}=1$, edad $=1$, nivel de instrucción = 1 y salario $=3$ ), conociendo el número total de jóvenes pertenecientes a estas categorías específicas ( 285 hombres, 224 jóvenes de edad comprendida entre 15 y 19 años, 227 jóvenes pertenecientes al nivel de educación primario y 85 jóvenes de salario comprendido entre 50.000 y 100.000 pts.), podríamos obtener las frecuencias marginales correspondientes y el valor esperado, según situación de independencia entre variables.

$$
f_{1113(\text { esperado })}=\left(\frac{f_{1000}}{f_{0000}} \cdot \frac{f_{0100}}{f_{0000}} \cdot \frac{f_{0010}}{f_{0000}} \cdot \frac{f_{0003}}{f_{0000}}\right) \times f_{0000}=\left(\frac{285}{542} \cdot \frac{224}{542} \cdot \frac{227}{542} \cdot \frac{85}{542}\right) 542=7.73
$$

En consecuencia, el valor de la relación entre las frecuencias observada y esperada es, en este caso particular, superior a la unidad 
(como puede apreciarse en el tercer registro de las combinaciones pertenecientes al subgrupo 2).

$$
R=\frac{\left.f_{1113(\text { observad })}\right)}{\left.f_{1113(\text { ssperad })}\right)}=\frac{11}{7.73}=1.42
$$

Mostramos, a continuación, las combinaciones más representativas, agrupadas según tipos, reducidos de la coherencia de la asociación de las variables.

1. Jóvenes de ambos sexos, edad inferior a 20 años, bajo nivel de instrucción, en paro.

\begin{tabular}{cccccc}
\hline Sexo & Edad & Estudios & Salario & Relación & N. ${ }^{\circ}$ jóvenes \\
\hline 1 & 1 & 1 & 1 & 1,39 & 57 \\
1 & 1 & 2 & 1 & 1,55 & 52 \\
2 & 1 & 1 & 1 & 1,22 & 47 \\
2 & 1 & 2 & 1 & 1,55 & 49 \\
\hline
\end{tabular}

2. Jóvenes trabajadores de ambos sexos, de edad inferior a 25 años, bajos niveles de instrucción y salario

\begin{tabular}{cccccc}
\hline Sexo & Edad & Estudios & Salario & Relación & N. $^{\circ}$ jóvenes \\
\hline 1 & 1 & 1 & 2 & 1,11 & 4 \\
2 & 1 & 1 & 2 & 2,06 & 7 \\
1 & 1 & 1 & 3 & 1,42 & 11 \\
1 & 2 & 1 & 3 & 2,08 & 17 \\
1 & 2 & 2 & 3 & 1,79 & 12 \\
\hline
\end{tabular}

3. Mujeres, de edad superior a 20 años, paradas.

\begin{tabular}{cccccc}
\hline Sexo & Edad & Estudios & Salario & Relación & N. $^{\circ}$ jóvenes \\
\hline 2 & 2 & 2 & 1 & 1,16 & 26 \\
2 & 2 & 3 & 1 & 1,65 & 17 \\
2 & 3 & 3 & 1 & 2,02 & 7 \\
\hline
\end{tabular}


4. Mujeres de edad superior a los 20 años, variado nivel de instrucción y salarios relativamente bajos.

\begin{tabular}{cccccc}
\hline Sexo & Edad & Estudios & Salario & Relación & N. $^{\circ}$ jóvenes \\
\hline 2 & 2 & 2 & 2 & 1,29 & 3 \\
2 & 2 & 3 & 2 & 1,56 & 1 \\
2 & 2 & 2 & 3 & 1,74 & 11 \\
2 & 2 & 3 & 3 & 1,73 & 3 \\
2 & 3 & 1 & 2 & 1,47 & 2 \\
2 & 3 & 1 & 3 & 1,62 & 6 \\
2 & 3 & 2 & 3 & 1,32 & 4 \\
2 & 3 & 3 & 2 & 3,27 & 1 \\
2 & 3 & 3 & 3 & 6,02 & 5 \\
\hline
\end{tabular}

5. Jóvenes trabajadores masculinos, de edad superior a 20 años, con bajo nivel de instrucción y salarios elevados.

\begin{tabular}{cccccc}
\hline Sexo & Edad & Estudios & Salario & Relación & N. $^{\circ}$ jóvenes \\
\hline 1 & 2 & 1 & 4 & 2,77 & 13 \\
1 & 2 & 1 & 5 & 1,93 & 4 \\
1 & 2 & 2 & 4 & 1,30 & 5 \\
1 & 3 & 1 & 4 & 3,11 & 7 \\
1 & 3 & 2 & 4 & 2,71 & 5 \\
1 & 3 & 2 & 5 & 4,94 & 4 \\
1 & 3 & 1 & 5 & 3,03 & 3 \\
\hline
\end{tabular}

6. Jóvenes trabajadores de ambos sexos, de edad superior a 20 años, alto nivel de instrucción y salario.

\begin{tabular}{cccccc}
\hline Sexo & Edad & Estudios & Salario & Relación & N. $^{\circ}$ jóvenes \\
\hline 1 & 3 & 3 & 5 & 22,45 & 5 \\
2 & 2 & 3 & 4 & 3,00 & 3 \\
2 & 3 & 3 & 5 & 14,27 & 3 \\
2 & 3 & 2 & 4 & 1,73 & 3 \\
2 & 3 & 3 & 4 & 10,47 & 5 \\
\hline
\end{tabular}


METODOLOGÍA DE TIPOS IDEALES CON DATOS CATEGÓRICOS...

\%. Estereotipos que contradicen los tipos anteriores.

\begin{tabular}{cccccc}
\hline Sexo & Edad & Estudios & Salario & Relación & N. $^{\circ}$ jóvenes \\
\hline 1 & 2 & 3 & 2 & 2,95 & 2 \\
1 & 3 & 3 & 3 & 1,14 & 1 \\
\hline
\end{tabular}

Los resultados obtenidos permiten deducir un importante conjunto de conclusiones:

- Únicamente un porcentaje de las combinaciones posibles entre las categorías (36 \%) manifiestan un grado de representatividad suficiente, como consecuencia de la asociación de las variables.

- En este limitado número de casillas, quedan incluidos el $73 \%$ de los jóvenes.

- Los 33 registros significativos pueden agruparse en seis tipos de jóvenes, que manifiestan una posición coherente frente a su inserción en el mercado laboral.

Interpretación de los datos de la encuesta sobre el mercado laboral juvenil, a partir de los tipos deducidos

La disponibilidad de una información, organizada por tipos de jóvenes con una problemática laboral homogénea, permite plantearse la posibilidad de un estudio por subgrupos o colectivos específicos. Esta forma de trabajo contrasta, positivamente, con el análisis del colectivo global, que exige el tratamiento de las variables, de manera individual o relacionada, incluyendo a grupos de personas muy heterogéneos. Además, el examen de los tipos, integrados por jóvenes con una problemática semejante, autoriza la inferencia de conclusiones válidas para el conjunto, circunstancia que facilita el dictamen de la situación y la adopción de medidas correctoras.

El tratamiento diferencial de la información por tipos exige la reorganización de la misma en ficheros aislados, válidos para cada uno de los grupos de individuos afines, deducidos de la selección pertinente de los registros del fichero general. Esta operación puede resultar laboriosa, si la extracción de los mismos no obedece a alguna regla de carácter ló-

$$
-451-
$$


gico. Sin embargo, la tarea merece la pena, ya que la descripción y el examen de los tipos resultantes ofrece un panorama de interpretación altamente significativo. La comparación de tablas de contingencia semejantes, correspondientes a los diversos subgrupos representativos del colectivo, otorga al estudio una concisión y rigor, difíciles de hallar al analizar el conjunto global de los datos. Además, la verificación de la relación existente entre variables, siguiendo un guión previamente establecido, permite contrastar de manera más eficiente las características de los «tipos» de jóvenes y su problemática laboral.

Los jóvenes más problemáticos (a).-La incorporación laboral del colectivo de jóvenes que abandonan la escuela entre los 16 y los 19 años es, sin duda, problemática. Salvo casos aislados, la relación con el mercado de trabajo viene definida por la inestabilidad. La posición más desfavorable es, lógicamente, la marginación por el desempleo. Muchos de los jóvenes parados pertenecen a la categoría de los que buscan el primer empleo. Su escasa o nula experiencia laboral y su deficiente preparación profesional (aunque posean un título de poco les sirve al no estar contrastado por la práctica) les dificulta, en gran manera, la posibilidad de encontrar trabajo.

Este colectivo está integrado por los jóvenes de ambos sexos, de edad inferior a 20 años, con un bajo nivel de instrucción, que se hallan en situación de paro (subgrupo 1). En total, superan, ligeramente, la mitad del conjunto de los jóvenes incluidos en los seis tipos de referencia. Constituyen, sin duda, el grupo más problemático. Su temprana separación del mundo educativo es la consecuencia de un fracaso escolar acentuado y tiene que ver más con el entorno social familiar que con dificultades económicas que obliguen al abandono (Cuadro II).

La dependencia de los padres es prácticamente total (el 97,6\% conviven con sus padres) y se halla influenciada por las desfavorables circunstancias de encontrar acomodo en el mercado laboral (el 66\% manifiesta como causa de dependencia el no tener trabajo).

La escasa edad de los jóvenes pertenecientes a este grupo, y su actual situación en paro, no es óbice para que un importante porcentaje del mismo haya tenido alguna experiencia laboral. De hecho, el $50 \%$ manifiesta haber realizado algún trabajo sin contrato (de ellos, el $45 \%$ en más de una ocasión) y únicamente el $17,8 \%$ con contrato legal (Cuadro III). 
CUADRo II

EDAD Y CAUSA DEL ABANDONO ESCOLAR

Jóvenes más problemáticos (a)

\begin{tabular}{lc}
\hline Edad de abandono & Porcentaje \\
\hline 14 años & 15,6 \\
15 años & 14,1 \\
16 años & 30,2 \\
17 años & 17,2 \\
18 años & 12,5 \\
18 años & 9,4 \\
& \\
Causa del abandono & \\
& \\
No me gustaba estudiar & 67,7 \\
Dificultades económicas familiares & 16,9 \\
No encontrar plaza & 3,1 \\
Otras & 12,3 \\
\hline
\end{tabular}

Fuente: E.P.

$$
\text { CUADro III }
$$

PORCENTAJE DE JÓVENES QUE TRABAJARON

Y NÚMERO DE VECES QUE LO REALIZARON

Jóvenes más problemáticos (a)

Sin contrato

\begin{tabular}{lcccccc}
\hline & $\mathbf{1}$ & $\mathbf{2}$ & $\mathbf{3}$ & $\mathbf{3}$ & N.C. & Total \\
\hline Sí & 27,2 & 10,3 & 7,1 & 5,4 & 12,0 & 62,0 \\
No & - & - & - & - & - & 38,0 \\
\hline
\end{tabular}

\section{Con contrato legal}

\begin{tabular}{lcccccc}
\hline & $\mathbf{1}$ & $\mathbf{2}$ & $\mathbf{3}$ & $>\mathbf{3}$ & $\mathbf{N . C}$ & Total \\
\hline Sí & 13,0 & 1,2 & 1,8 & 1,8 & - & 17,8 \\
No & - & - & - & - & - & 82,2 \\
\hline
\end{tabular}

FuENTE: E.P. 
El hallar el primer trabajo difícilmente rompe el círculo vicioso inexperiencia-desempleo. La adquisición de una experiencia profesional, sea cual sea ésta, resulta la mayoría de las veces traumática y no siempre enriquecedora, en la perspectiva de construirse un currículum profesional valorado socialmente. Gran parte de los primeros trabajos son poco atractivos y de reducida duración, lo que genera desánimo y falta de expectativas laborales. La gran rotación del empleo juvenil ha sido destacada como una de las características fundamentales del trabajo en este grupo específico. La principal explicación hay que buscarla en la naturaleza de los empleos que se ofrecen a los adolescentes, dado que muchos de ellos no tienen porvenir y consisten en tareas subalternas y poco formativas (Cuadro IV).

Otras salidas profesionales completan el panorama de la actividad laboral de estos jóvenes. Una de las más usuales es la denominada «ayuda familiar», trabajando con un familiar, sin que ello suponga recibir una retribución garantizada (el 35,5\% de estos jóvenes se hallaba realizando un trabajo de estas características). Este residuo de la economía precapitalista, poco o nada ayuda al proceso de emancipación juvenil y suele constituir un refugio en época de crisis.

A pesar de la elevada tasa de rotación laboral, muchos jóvenes no cesan en su empeño de búsqueda de un nuevo trabajo. A esta temprana edad, la disponibilidad de un dinero propio, que les permita una cierta independencia económica, resulta una motivación superior que la preparación de cara a un trabajo más estable y duradero (Cuadro V). En este sentido, un porcentaje elevado de los encuestados manifestaban su disposición a aceptar trabajos en precario.

Los jóvenes más problemáticos (b).--Pertenecen a este colectivo los jóvenes trabajadores de ambos sexos, de edad inferior a 25 años, con bajos niveles de instrucción, ocupados y con reducido salario (subgrupo 2). En realidad, este grupo podría haber sido incluido en el anterior, ya que su inserción en el mercado de trabajo puede considerarse, en la mayoría de los casos, como circunstancial; fruto del movimiento de vaivén, característico de la rotación laboral que afecta a los empleos de bajo nivel profesional.

Estos jóvenes forman parte fundamental del mercado laboral secundario, de empleos inestables y mal remunerados, con escasas o nulas posibilidades de superación personal, consecuencia del bajo nivel de 
CuAdro IV

PRINCIPALES TRABAJOS DESEMPEÑADOS EN LA ETAPA

DE OCUPACIÓN

Jóvenes más problemáticos (a)

\begin{tabular}{lc}
\hline Tipo de ocupación & Porcentaje \\
\hline Personal no cualificado & 55,3 \\
Peones & 15,5 \\
Vendedores & 20,0 \\
Obreros construcción & 8,2 \\
\hline
\end{tabular}

Fuente: E.P.

CUAdro V

PORCENTAJE DE JÓVENES EN BÚSQUEDA DE TRABAJO Y MOTIVO PARA NO BUSCAR. DISPOSICIÓN A ACEPTAR UN TRABAJO

\begin{tabular}{|c|c|c|}
\hline Motivo para no buscar & Sí & No \\
\hline Falta de preparación & - & 3,6 \\
\hline No lo necesita & - & 9,2 \\
\hline Desencanto & - & 1,0 \\
\hline No compensa económicamente & - & - \\
\hline Otros & - & 7,7 \\
\hline n.c. & - & 3,6 \\
\hline TOTAL & 75,0 & 25,0 \\
\hline Disposición a trabajar & \multicolumn{2}{|c|}{ Porcentaje } \\
\hline Cualquier trabajo & \multicolumn{2}{|c|}{49,0} \\
\hline Movilidad geográfica & \multicolumn{2}{|c|}{22,5} \\
\hline Cualquier tipo de contrato & \multicolumn{2}{|c|}{9,5} \\
\hline Sueldo bajo & \multicolumn{2}{|c|}{12,0} \\
\hline Otros & \multicolumn{2}{|c|}{7,0} \\
\hline
\end{tabular}

Fuente: E.P.

formación adquirido. En realidad, esta circunstancia se pone de manifiesto si observamos alguna de las características que definen su posición en el mercado de trabajo: 
- Bajo nivel de formación (el 61,1\% no supera el nivel de F.P.1).

- Prematuro abandono del sistema educativo reglado (el 67,5\% antes de los 16 años).

- Alto grado de insatisfacción con los estudios realizados (el $50 \%$ manifiesta no haberles servido para nada).

- Bajos salarios y elevado índice de temporalidad (únicamente el $16,7 \%$ tenían empleos fijos).

- Empleos caracterizados por su escaso nivel de especialización (Cuadro VI).

- La mayor parte continúa viviendo con sus padres (el 88,9\%) y un elevado número de ellos manifiestan que la causa fundamental es por motivos económicos (el 76\%).

CUADRO VI

PRINCIPALES TRABAJOS DESEMPEÑADOS

Jóvenes más problemáticos (b)

\begin{tabular}{lc}
\hline Tipo de ocupación & Porcentaje \\
\hline Personal no cualificado & 25,0 \\
Obreros sin especialización & 25,0 \\
Vendedores & 22,9 \\
Obreros especializados & 20,8 \\
Administrativos & 6,3 \\
\hline
\end{tabular}

Fuente: E.P

Las mujeres. - El modelo de integración laboral femenina ha sufrido una modificación trascendental en los últimos tiempos. Durante los primeros años setenta, la incorporación de la mujer al trabajo se producía, mayoritariamente, en el escalón de edad comprendido entre los 30 y los 45 años. El adelanto de la matemidad, junto con la notable extensión de la enseñanza pública de sus hijos y la tecnificación del trabajo doméstico, permitió ya una reincorporación al mercado de trabajo a una edad más temprana. Sin embargo, el cambio más drástico se ha producido durante los últimos quince años. La mujer, de edad comprendida en- 
tre los 20 y los 35 años, ha hecho subir las tasas de actividad, en muchos casos, independientemente del cambio de estado civil.

Además, el colectivo femenino mantiene sus puestos de trabajo en condiciones bien diferentes, con la mejora de sus ocupaciones. Hasta hace poco tiempo, el trabajo de la mujer se centraba en la ayuda familiar en la agricultura, el comercio, la hostelería, el servicio doméstico y determinadas ramas industriales como la alimentación y el textil. Hoy en día, el nivel profesional de la mujer ha sufrido un notable incremento, lo que se ha visto reflejado en el gran número de tituladas medias y superiores, que vienen desarrollando su profesión, fundamentalmente en el sector servicios, en las ramas de la educación, la sanidad y la administración pública en general. Tres elementos fundamentales han influido en este cambio decisivo: la radical disminución del número de hijos por mujer, la igualación del nivel de estudios por sexo y el mantenimiento de la ocupación de la mujer con el matrimonio, e, incluso, con la maternidad (Garrido, L. J. y Toharia, L.; 1991).

Se considera aquí, exclusivamente, el colectivo de mujeres de posición intermedia en el mercado laboral, ya que la problemática de las más jóvenes y la de las tituladas universitarias bien situadas (que analizaremos con posterioridad) coincide en gran parte con el de los varones. Podemos incluir en este segmento específico de la población trabajadora a dos colectivos, que hemos separado por su diferente situación respecto a la actividad profesional: paradas (subgrupo 4) y ocupadas (subgrupo 5).

Tanto unas como otras poseían un aceptable nivel de formación (Cuadro VII), que no se correspondía, sin embargo, con su ubicación en el mercado de trabajo. Resulta significativo, que el $50 \%$ de las mujeres de edad comprendida entre los 20 y los 29 años se encontrara en el paro. A pesar de su fracaso laboral, una gran parte de este colectivo se hallaba en busca de trabajo (el 90,7\%) y manifestaba estar dispuesto a aceptar cualquier tipo de empleo (el 67,4\%). La experiencia laboral anterior (Cuadro VIII) muestra el elevado porcentaje de los empleos ilegales y temporales (el 59,1\% de las mujeres expresó como causa de la perdida de empleo la rescisión de su contrato) y revela, a su vez, la elevada rotación laboral de un colectivo social altamente discriminado.

Por su parte, el colectivo de mujeres ocupadas trabaja mayoritariamente en el sector servicios (el 88,2\%), en variadas categorías pro-

$$
-457-
$$


Cuadro VII

NIVEL DE INSTRUCCIÓN (PORCENTAJES)

Mujeres

\begin{tabular}{lcc}
\hline Nivel de instrucción & Paradas & Ocupadas \\
\hline F.P. 1 & 0,0 & 26,7 \\
B.U.P.-F.P. 2 & 24,4 & 30,0 \\
C.O.U. & 13,3 & 20,0 \\
Universitario & 62,3 & 23,3 \\
\hline
\end{tabular}

Fuente: E.P.

CUADro VIII

PORCENTAJE DE JÓVENES QUE TRABAJARON

Y NÚMERO DE VECES QUE LO REALIZARON

Mujeres

\begin{tabular}{lcccccc}
\hline & $\mathbf{1}$ & $\mathbf{2}$ & $\mathbf{3}$ & $\mathbf{> 3}$ & N.C. & Total \\
\hline Sin contrato & & & & & & \\
$\quad$ Sí & 28,9 & 11,1 & 2,2 & 11,1 & 6,7 & 60,0 \\
$\quad$ No & - & - & - & - & - & 40,0 \\
Con contrato legal & & & & & & \\
$\quad$ Sí & 20,5 & 17,1 & 4,9 & 7,3 & 4,9 & 53,7 \\
No & - & - & - & - & - & 46,3 \\
\hline
\end{tabular}

Fuente: E.P.

CUADRo IX

PRINCIPALES TRABAJOS DESEMPEÑADOS

Mujeres

\begin{tabular}{lc}
\hline Tipo de ocupación & Porcentaje \\
\hline Vendedoras & 37,0 \\
Admistrativos & 25,9 \\
Personal no cualificado & 14,9 \\
Técnicos superiores & 7,4 \\
Encargados & 7,4 \\
Agentes comerciales & 7,4 \\
\hline
\end{tabular}

Fuente: E.P. 
fesionales (Cuadro IX), con reducidos niveles de salario (el 19,4\%, menos de 50.000 pts. y el resto, inferior a las 100.000 pts.) y altas tasas de temporalidad (únicamente el 17,2\% disponían de empleo fijo), lo que evidencia, igualmente, la postergación laboral de la mujer, circunstancia doblemente lesiva en el caso de las mujeres jóvenes.

Los jóvenes de procedencia obrera.-La discriminación por sexo, en materia laboral, se pone, de nuevo, en evidencia ante la presencia de un subgrupo de características simétricas al anterior: los trabajadores jóvenes varones, mayores de 20 años, con bajo nivel formativo y salarios relativamente más altos (subgrupo 5).

El denominado «modelo obrero», como prototipo de la independencia juvenil en el medio proletario, quedaba definido, en el varón, por el paso casi instantáneo de la infancia a la edad adulta y la concordancia en el tiempo del desarrollo activo de la vida profesional, el matrimonio y el abandono del hogar paterno. Hoy en día, el modelo ha desaparecido en sentido estricto, pero conserva algunos rasgos específicos, sobre todo los derivados del abandono de la escuela y la incorporación al mundo del trabajo. En muchas familias obreras, la integración a corta edad en la «fábrica» o en el «tajo» es valorada positivamente, al adivinar en la continuidad de los estudios una doble perdida: la de adquirir una experiencia profesional y la de ingresar los emolumentos económicos generados por el trabajo.

Este colectivo de jóvenes revela, en gran parte, su procedencia de esta clase social. Constituye un subgrupo homogéneo, en cuanto a la formación y categoría profesional de los padres se refiere (ninguno supera la formación de grado medio y un número muy elevado pertenece a los obreros con o sin especialización) y su nivel de estudios (Cuadro X) refleja su temprano abandono del sistema educativo (el 89\%, antes de los 20 años) y su pronta incorporación al mundo laboral (el $86 \%$, antes de los 20 años).

Los trabajos desempeñados se encuentran repartidos por los tres principales sectores productivos, aunque superan, ampliamente, frente a los demás colectivos, su participación en el sector secundario (el $51,2 \%$ trabajan en la industria y la construcción). La estructura profesional destaca por el peso relativo de las profesiones de obreros con diverso grado de especialización (el 68,4\% pertenece a estas categorías profesionales) (Cuadro XI). 
Cuadro X

NIVEL DE INSTRICCIÓN

Jóvenes de procedencia obrera

\begin{tabular}{lc}
\hline Nivel de instrucción & Porcentaje \\
\hline F.P. 1 & 56,1 \\
B.U.P.-F.P. 2 & 31,7 \\
C.O.U. & 7,3 \\
Universitario & 4,9 \\
\hline
\end{tabular}

Fuente: E.P.

CUAdro XI

PRINCIPALES TRABAJOS DESEMPEÑADOS

Jóvenes de procedencia obrera

\begin{tabular}{lc}
\hline Tipo de ocupación & Porcentaje \\
\hline Obreros especializados & 34,2 \\
Obreros sin especializar & 26,3 \\
Dependientes & 10,6 \\
Administrativos & 7,9 \\
Personal no cualificado & 7,9 \\
Agentes comerciales & 5,3 \\
Director de empresa & 2,6 \\
Mando intermedio & 2,6 \\
Técnico graduado medio & 2,6 \\
\hline
\end{tabular}

Fuente: E.P.

En realidad, este colectivo se halla integrado por jóvenes, que a pesar de su escaso nivel de instrucción valoran positivamente su pertenencia al mundo del trabajo: por el sueldo que reciben y por el orgullo por el oficio desempeñado. No es extraño que un apreciable porcentaje (el $41,4 \%$ ) posean contratos fijos y que todos superen el sueldo de 100.000 pts. (el 26,9 superan las 150.000 pts.), manifestando ampliamente su satisfacción por el trabajo realizado (el $69 \%$, mucho o bastante, frente al $9,5 \%$, poco o nada). 
La mayoría (el 95\%) expresan su alto grado de independencia económica, aunque un estimable porcentaje continúan viviendo con sus padres, más por el estilo de vida que por la imposibilidad económica de emanciparse.

\section{Los titulados universitarios, bien colocados en el mercado laboral}

El segmento de la población juvenil que ocupa una posición privilegiada en el mercado laboral, por mor de su superior nivel de instrucción, es el de los titulados medios y superiores. La situación ha cambiado, sin embargo, de la existente durante los años setenta, cuando la educación universitaria era una ruta segura para obtener un buen empleo, mientras en la actualidad no podemos dejar de advertir un panorama sombrío de salarios caídos y escasas oportunidades profesionales.

Pero a pesar de ello, la demanda en educación superior ha aumentado en todos los países (particularmente en el nuestro), por lo que la consecución de un título sigue siendo una opción razonable, ya que los hechos son tozudos al mostrar una panorámica laboral más alentadora para este estrato de población, con unas tasas de actividad más elevadas y unas tasas de paro inferiores al del resto de la población. Esta posición favorable ha sido sustancial, sobre todo, en la fase de recuperación de la economía, lo que muestra la mejoría de expectativas de trabajo de los titulados, en el proceso de reorganización productiva que está teniendo lugar los últimos años. La explicación del diferente posicionamiento ante el mercado laboral, entre titulados y resto de la población, puede girar en torno a tres hipótesis. Por un lado, y por lo que refiere al sector productivo, la reestructuración industrial (reconversión, cambio tecnológico, etc.) conllevaría un mayor aumento relativo del personal cualificado (titulados medios y superiores) respecto al poco cualificado. En segundo lugar, la política de creación de empleo público, en particular a partir de 1982, ha hecho posible la incorporación a este mercado de trabajo específico a un importante contingente de titulados jóvenes. Finalmente, la ocupación, por parte de algunos jóvenes con nivel de instrucción superior, de empleos que requieren menor cualificación, desplazando a los activos menos cualificados (Consejo de universidades; 1989). Esta situación de subempleo de los titulados supe- 
riores es tanto más importante, en cuanto que no parece ser un fenómeno coyuntural ligado a una recesión económica pasajera, sino todo un síntoma de una sociedad sobreescolarizada.

Los jóvenes pertenecientes al estrato más privilegiado (alto nivel de instrucción, desarrollando un trabajo con los salarios relativos más elevados) (subgrupo 6) constituyen un «ave raris», ya que únicamente el $3,5 \%$ del total del colectivo total están integrados en el mismo.

Su posición preeminente se corresponde con la independencia económica respecto a los padres (el 89,2\% se manifestaba económicamente independiente), aunque un porcentaje apreciable de los mismos, sobre todo los solteros, continúan viviendo en el domicilio paterno (el 42,1\%). El hogar familiar, a pesar de que las relaciones son superficiales y poco profundas, se percibe como grato y permisivo. Un número apreciable de jóvenes lo prefieren a la vida independiente o en pareja, incluso cuando se aproximan a la edad de treinta años.

La mayoría de los jóvenes encuestados, pertenecientes a este grupo, se muestran satisfechos con los estudios realizados y se adivina en sus respuestas que la elección de la carrera desarrollada se halla en consonancia con los deseos de alcanzar un nivel profesional específico (el 47,4\% mucho o bastante, frente al 23,0\%, poco o nada).

Además del alargamiento del periodo escolar, la entrada en la vida activa suele tener lugar de forma progresiva y no instantánea, como se producía hace años. El tipo de jóvenes que nos ocupa pertenece, sin embargo, a un grupo privilegiado, por cuanto no ha tenido que esperar demasiado tiempo para encontrar un trabajo, en muchos casos, adecuado a sus aspiraciones, circunstancia que se observa en su limitada experiencia laboral anterior, tanto en ocupaciones sin contrato de trabajo, como en otras situaciones laborales legales (únicamente el 15,0\% y el 20,8\%, respectivamente, manifiestan haber tenido una experiencia laboral sin contrato o con contrato legal).

Los jóvenes universitarios abandonan el sistema educativo reglado con un nivel de formación relativo superior y se hallan en una posición competitiva más favorable, en muchos casos, de encontrar un trabajo acorde con su titulación. Así, aparece una correlación altamente positiva entre la titulacion universitaria alcanzada y el tipo de ocupación que desempeñan los jóvenes integrados en este colectivo específico, aunque un porcentaje nada desdeñable de los mismos se ve obligado a aceptar determinados puestos de trabajo, que tienen muy poco que ver con la 
preparación recibida, produciéndose el efecto expansión hacia los estratos de menor nivel profesional en la estructura ocupacional (Cuadro XII).

CUADRO XII

OCUPACIÓN DESEMPEÑADA

Titulados universitarios, bien situados

\begin{tabular}{lc}
\hline Ocupación & Porcentaje \\
\hline Técnicos superiores & 16,7 \\
Técnicos medios & 27,8 \\
Encargados & 5,6 \\
Agente comercial & 10,2 \\
Vendedor & 5,6 \\
Administrativo & 5,6 \\
Obrero especializado & 16,8 \\
Obreros no cualificados & 10,2 \\
\hline
\end{tabular}

Fuente: E.P.

La mayor cualificación profesional relativa de la mano de obra que integra este grupo explica que un porcentaje importante del mismo ejerza su labor profesional en el sector terciario (el 68,8\%), el sector productivo que absorbe el mayor porcentaje de titulados, frente al $20,8 \%$ que trabajan en la industria y el 10,4\% en la construcción.

La flexibilidad de la actividad laboral y el cambio radical de la estructura empresarial del momento presente han contribuido a la segmentación del mercado laboral en submercados de características contrastadas. Frente al mercado laboral secundario, existiría un mercado de trabajo primario, de mejores salarios, empleos estables y amplias posibilidades de promoción profesional. Un porcentaje importante de los jóvenes pertenecientes a este tipo específico se hallaría integrado en este submercado de trabajadores privilegiados, ya que además de poseer empleos bien pagados (relativamente, los más elevados), dispondrían de una superior estabilidad en el empleo (el 76,9\% trabajarían con contratos fijos, frente al 33,1\% de eventuales). No es extraño, por tanto, que la mayor parte de los mismos se manifiesten satisfechos con el trabajo que re-

$$
-463-
$$


alizan (el 63,2\% se mostraron bastante o muy satisfechos y únicamente el $15,7 \%$ poco o nada).

\section{Conclusiones}

La utilización de datos personales, no agregados a ningún nivel de la escala espacial, permite la definición de «tipos», mediante el procedimiento de clasificar aquellos elementos de un colectivo de estudio, que resulten más significativos. La comparación de los valores obtenidos en la distribución de la información, en una tabla de contingencia multidimensional, según las dimensiones fundamentales del fenómeno a analizar, respecto a los de la hipótesis de independencia entre variables, se presenta como una vía de investigación aceptable para descubrir e identificar los individuos más representativos del mismo.

La aplicación del método descrito al análisis del mercado laboral juvenil, a partir de un conjunto de variables básicas: sexo, edad, nivel de instrucción, relación con la actividad económica (ocupado/parado) y salario, ofrece una interesante perspectiva metodológica de cómo agrupar el colectivo de jóvenes, de edad comprendida entre 15 y 29 años, que han abandonado el sistema educativo reglado, en «tipos» homogéneos respecto a la problemática de su inserción en el mundo del trabajo. La identificación de varios subtipos, con personalidad definida, sirve de ejemplo de cómo analizar un conjunto de personas heterogéneo por los rasgos definitorios de los arquetipos que lo integran.

\section{BIBLIOGRAFÍA}

Agresti, A. (1990): Categorical Data Analysis. Jhon Wiley Sons. New York, 557 pp. Aguilera Arilla, M. J.; Azcárate Luxán, M. V.; Borderías Uribeondo, M. P.; González YanCI, M. P.; Murillo Castillejo, J. y Santos Preciado, J. M. (1996): Desequilibrios del mercado de trabajo juvenil en el A. M. M. Informe Técnico presentado a la Dirección General de la Juventud de la Consejería de Educación y Cultura de la C.A.M. Madrid, 527 pp.

Bosque Sendra, J.; Chuvieco Salinero, E. y Santos Preciado, J. M. (1983): «Algunos problemas metodológicos de las técnicas cuantitativas en Geografía Humana». La Geografía Teórica y Cuantitativa. Concepto y método. I Coloquio de Geografía Cuantitativa. Oviedo, pp

CASAL, J.; Masjuán, J. M. y Planas, J. (1991): La inserción social y profesional de los jóvenes. CIDE, Ministerio de Educación y Ciencia. Madrid, 337 pp. 
CONSEJO DE UNIVERSIDADES (1989): El stock de titulados universitarios y su relación con el mercado de trabajo, 1976-86, Ministerio de Educación y Ciencia. Madrid, 251 pp.

EusYa (1988): Paro y empleo juvenil en la periferia urbana madrileña. Plan de Prevención de la Delincuencia y la Marginación Social en San Blas, Orcasitas y Pan Bendito. Madrid, $231 \mathrm{pp}$.

Figuera GAZo, P. (1996): La inserción del universitario en el mercado de trabajo. EUB, S. L. Barcelona, 332 pp.

GARRIDO, L. J. y TOHARIA, L. (1991): Prospectiva de las ocupaciones y la formación en la España de los noventa. Instituto de Estudios y Análisis Económicos, Ministerio de Economía y Hacienda. Madrid, 233 pp.

JimÉnEZ BLANCO J. (1978): «Weber, Schutz y Garfinkel sobre racionalidad» en JiMÉNEZ Blanco, J. y Moya Valgañón, C. Teoría Sociológica Contemporánea. Tecnos. Madrid, pp 366-396.

Muguruza Cañas, C. y Santos Preciado, J. M. (1989): «La importancia de las unidades de análisis en el modelo de la ecología factorial». Espacio Tiempo y Forma. Serie VI, Geografía, t. 2. U.N.E.D. Madrid, pp 87-102.

O.C.D.E. (1987): La naturaleza del desempleo de los jóvenes. Ministerio de Trabajo y Seguridad Social. Madrid, $320 \mathrm{pp}$.

Openshaw, S. (1981): «Le probleme de l'agregation spatiale en Géographie». L'Espace Geographique. París, pp 15-24.

Peinado López, A. (1988): La discriminación de la mujer en el mercado de trabajo espanol. Ministerio de Trabajo y Seguridad Social. Madrid, 198 pp.

Ruiz-Maya Pérez, L.; Martín Pliego, J.; López Ortega, J.; Montero Lorenzo, J. M. y Uriz Tomé, P. (1990): Metodología estadística para el análisis de datos cualitativos. C.I.S. Madrid, 382 pp.

SÁNCHEZ CARRIÓN, J. J. (1984): Introducción a las técnicas de análisis multivarieble aplicadas a las ciencias sociales. C.I.S. Madrid, $331 \mathrm{pp}$.

RESUMEN: El análisis de datos cualitativos, comparando la distribución de la información de una tabla de contingencia múltiple, respecto a la hipótesis de independencia de las variables, permite la construcción de «tipos ideales», como método de interés para analizar una problemática socio-territorial concreta. La definición y posterior aplicación de esta alternativa metodológica al estudio de la incorporación de los jóvenes al mundo del trabajo revela la existencia de segmentos homogéneos en este específico mercado laboral.

ABSTRACT: The analysis of the qualitative data, comparing the distribution of the information in a table of contingency multiple with respect to the hypothesis of independence of the variables, permits the construction of «ideal types», as an interesting method to analize specific socio-territorial problems. The definition and subsequent application of this alternative methodology to the study of the incorporation of young people to employmen reveals the existence of homogeneous segments in this specific labour market. 